Revista de Matemática: Teoría y Aplicaciones 2012 19(1) : 103-106

CIMPA - UCR ISSN: 1409-2433

\title{
FACTORES QUE INCIDEN EN LA INVERSIÓN PRIVADA EN EL SALVADOR
}

\author{
INFLUENCE FACTORS IN PRIVATE \\ INVESTMENT IN EL SALVADOR
}

René Palacios* Nerys Funes ${ }^{\dagger}$

Received: 18-Feb-2010; Revised: 31-Mar-2011 \&3 8-Oct-2011;

Accepted: 28-Oct-2011

\begin{abstract}
Resumen
Según muchos estudios empíricos, uno de los determinantes fundamentales del ciclo económico de corto y mediano plazo es la inversión en bienes de capital, ya que forma parte de la demanda agregada, por lo que explica los cambios de fase y amplitud del ciclo productivo de una economía. En esta investigación se establecen los factores determinantes de la inversión privada en El Salvador. El estudio se hizo con base a registros económicos históricos del periodo 1958-2008, utilizando técnicas de regresión dinámica de series temporales. La metodología implementada es la siguiente: Se hace un análisis univariante de cada serie, con el objeto de identificar adecuadamente
\end{abstract}

*Escuela de Matemática, Facultad de CC. NN. y Matemática, Universidad de El Salvador, San Salvador. Final 25 Av. Norte y Calle San Antonio Abad, San Salvador, El Salvador. E-mail: jpalaciosb2000@yahoo.es

${ }^{\dagger}$ Misma dirección que/Same address as R. Palacios. E-Mail: nerys_funest@yahoo.com.mx 
el modelo ARIMA a que correspondan y se ajusta el modelo de regresión dinámico para la inversión privada. El objetivo de este estudio es contribuir al establecimiento de políticas económicas para El Salvador y brindar orientaciones al modelaje de fenómenos en los que intervienen series temporales.

Palabras clave: Correlaciones cruzadas, Modelos ARIMA, función de transferencia, estimación.

\begin{abstract}
According to many empirical studies, one aspect that determines the foundations of economic cycles in the short and medium term is the investment of capital goods since it belongs to an aggregate demand and explains the changes in phase and scope of the productive cycle in the economy. Here we establish factors that are determinant of the private investment in El Salvador. This study was prepared based on historic economic records from the period 1958-2008 and using dynamic regression techniques of time series. The methodology implemented is the following: A univariate analysis is undertaken of each series, with the goal of adequately identifying a proper ARIMA model that corresponds and adjusts to the dynamic regression model for the private investment. The objective of this study is to contribute to the establishment to the economic policies for El Salvador and to offer orientation for the modeling of phenomena in which time series are involved.
\end{abstract}

Keywords: Cross Correlation, ARIMA Model, transfer function, estimation.

Mathematics Subject Classification: 62M10.

\title{
1 Ajuste del modelo de regresión dinámica
}

\subsection{Análisis univariante}

Se hace un análisis descriptivo de las series: préstamos disponibles para inversión (prestamos1990), exportaciones (exptot1990) e importaciones totales (imptot1990), deuda externa (deuda_ext1990), PIB: producto interno bruto (pibconst1990), inversión pública (inv_pub2009) y privada (inv_priv1990), IPC (índice de precios al consumidor), tasa de inflación (inflacion), tasa de crecimiento económico (TCE) y tasa de interés real (interes_real). Esto comprende hacer un análisis de los gráficos de secuencias de las series temporales y sus correlogramas, para luego estimar los modelos univariantes de cada una de las series. Los modelos ajustados a cada serie temporal son los siguientes: inv_priv1990 modelo 
$\operatorname{ARIMA}(0,1,0)$; inv_pub1990 modelo ARIMA(1,0,0); prestamos1990 modelo $\operatorname{ARIMA}(0,1,0)$; TCE modelo $\operatorname{ARIMA}(1,0,0)$; pibconst 1990 modelos $\operatorname{ARIMA}(1,1,0)$; interes_real modelo $\operatorname{ARIMA}(1,0,1)$; exptot1990 modelo $\operatorname{ARIMA}(0,1,1)$ y imptot1990 modelo $\operatorname{ARIMA}(0,1,0)$. Es de mencionar que algunos modelos contienen variables ficticias que corresponden a datos atípicos.

\subsection{Análisis Correlacional y modelo de regresión dinámica}

Se determinó la relación lineal existente entre todos los pares de variables posibles, con el fin de determinar las variables que van a participar en el modelo y determinar los posibles modelos alternativos. Con las correlaciones cruzadas también es factible establecer la dirección de causalidad de la correlación, es decir, si la correlación es en un sólo sentido o bidireccional. El modelo que se ajustó a la serie temporal de la inversión privada es un modelo ARIMA llamado modelo de función de transferencia. Básicamente es un modelo de regresión en el que las variables exógenas o explicativas son series de tiempo, las cuales se espera que den explicación a la variable inversión privada. Se realizó un análisis de residuos, para garantizar un correcto ajuste. El software que se utilizó fue el paquete estadístico GRETL por las bondades que éste ofrece. El modelo final ajustado, luego de depurar las variables que no resultaron significativas es el siguiente:

$$
\begin{aligned}
& \left(1-\text { L)inv_priv } 1990_{t}=\right. \\
& \quad 0.28 \text { Dpibconst } 1990_{t}-0.14 \text { Dpibconst } 1990_{t-1}-0.14 \text { Dpibconst } 1990_{t-2} \\
& \quad+0.51 \text { inv_pub1990 } 0_{t-1}-0.52 \text { inv_pub } 1990_{t-2}+0.24 \text { Dimptot } 1990_{t}
\end{aligned}
$$

donde Dpibconst $1990_{t}=$ pibconst $1990_{t}-$ pibconst $1990_{t-1}$, de igual forma se interpreta Dimptot1990, y $(1-L) X_{t}=X_{t}-X_{t-1}$.

La salida o resultado final que proporcionó el paquete GRETL, de la cual se obtuvo el modelo anterior, se muestra a continuación: El modelo final ARIMA, usando las observaciones 1961-2008 $(T=48)$ con el criterio de Máxima Verosimilitud, empleando como variable dependiente: (1 L)inv_priv1990 y desviaciones típicas basadas en la matriz de productos externos, se muestra en la tabla 1.

Observamos que se ha obtenido un modelo con todos los coeficientes significativos. Además el modelo tiene los menores valores de los estadísticos asociados a los criterios de selección de modelos, acorde a la teoría referida y ha soportado las pruebas residuales respectivas. 
Tabla 1: Modelo final ARIMA, con $(1-L) i n v \_p r i v 1990$ como variable dependiente y usando el paquete GRETL.

\begin{tabular}{|c|c|c|c|c|c|}
\hline Variables & Coef. & D. Típ. & Estad. $z$ & Valor $p$ & \\
\hline d_pibconst1990 & 0.27619 & 0.05376 & 5.1373 & $<0.00001$ & $* * *$ \\
\hline$d_{-}$pibconst $1_{1}$ & -0.14149 & 0.05986 & -2.3634 & 0.01811 & $* *$ \\
\hline$d_{-}$pibconst $1_{2}$ & -0.14462 & 0.05265 & -2.7469 & 0.00602 & $* * *$ \\
\hline inv_pub1990 & 0.50978 & 0.17595 & 2.8973 & 0.00376 & $* * *$ \\
\hline inv_pub1990 & -0.51857 & 0.17026 & -3.0458 & 0.00232 & $* *$ \\
\hline d_imptot 1990 & 0.23856 & 0.04964 & 4.8055 & $<0.00001$ & $* * *$ \\
\hline
\end{tabular}

Media de la vble. dep. $=20.300$

D.T. de la vble. dep. $=85.879$

media innovaciones $=1.9167$

D.T. innovaciones $=47.664$

Log-verosimilitud $=-253.589$

Criterio de Akaike $=521.179$

Criterio de Schwarz $=534.278$

Crit. de Hannan-Quinn $=526.129$

$\mathrm{SCR}=109,050$

\section{Conclusiones}

El PIB, las importaciones totales y la inversión pública son los factores que más influyen en la inversión privada en El Salvador. Por ello, se debe promover el desarrollo de estos aspectos en las políticas económicas, dado que no se puede negar la importancia de la inversión privada en el crecimiento económico del país.

\section{Referencias}

[1] Espasa, A.; Canceló, J.R. (1993) Métodos Cuántitativos para el Análisis de la Cuyuntura Económica, 1a edición. Alianza Editorial, Madrid.

[2] Hamilton, J.D. (1994) Time Series Analysis. Princeton University Press, Princeton NJ.

[3] Parkin, M.; Esquivel, G. (2001) Introducción a la Economía, un enfoque latinoamericano, 5a edición. Addison Wesley, México D.F.

[4] Peña, D. (2005) Análisis de Series Temporales, 1a edición. Alianza Editorial, Madrid. 